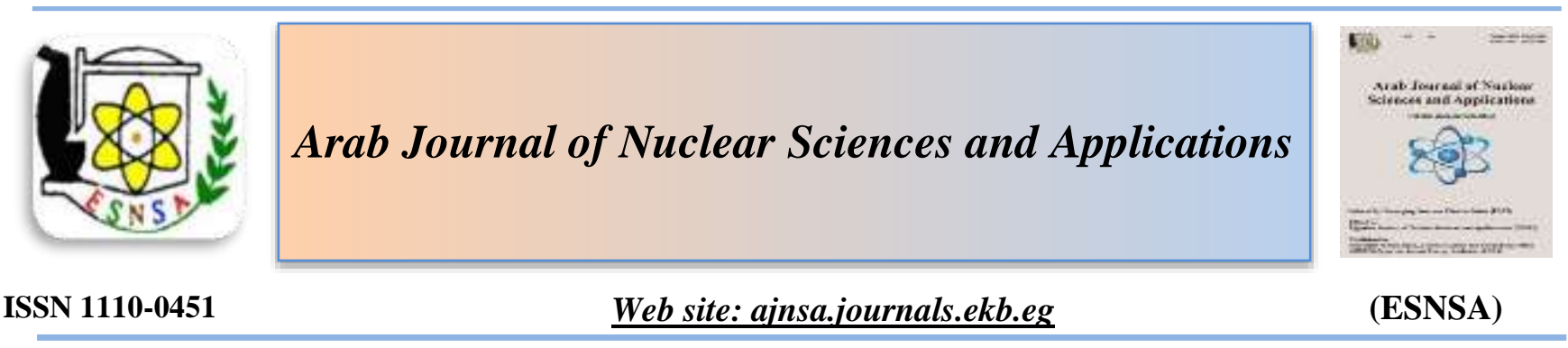

\title{
Study the Properties of 6061 Aluminum Alloy which used as Cladding for Nuclear Reactor Fuel by Using Positron Annihilation Doppler Broadening Technique
}

\author{
M. H. Abd-Elhakim**, M. Darweesh**, M. Abdel-Rahman*, M. A. Abdel-rahman* and Emad A. \\ Badawi* \\ *Physics Department, Faculty of Science, Minia University, Egypt \\ **Nuclear and Radiological Regulatory Authority
}

Received 26 ${ }^{\text {th }}$ Nov. 2019 Doppler - broadening spectroscopy is a technique that is used to know the properties of matter by Accepted $19^{\text {th }}$ May2020 means of gamma quanta. The entire energy of an annihilation positron - electron pair includes the energy according to its mass (= 2 x $511 \mathrm{Kev})$ and kinetic energy corresponding to the motion of the particles causing an energy shift of the annihilation line. The line shape has two parameters $S$ and $W$, but the $\mathrm{W}$ parameter is more sensitive to the chemical surrounding of the annihilation site than the $S$ parameter, because the core electron having high momentum are contributing. Mainlyin the region of large deviations from the annihilation energy of $511 \mathrm{kev}$. Both the $S$ and w parameter are sensitive to the concentration and kind of defect.The goal of our work is to investigate the dependence of annihilation parameters on crystal defect the content of $\mathrm{Mg}$ atoms in a series of commercial $\mathrm{Al}$ - alloys by Doppler broadening technique. Position parameter like (crystallite size - trapping rate - defect density dislocation density Storedenergy).

Keywords: AA 6061, Doppler - broadening spectroscopy, S and w parameter,trapping rate.

\section{Introduction}

Alloys 6061 is one of the most widely used alloys in 6000 series. It contains magnesium and silicon as its major elements. This standard structural alloy, one of the most versatile of the heat treatable alloys is popular for medium to high strength requirements and has good toughness characteristics. Applications range from transportation components to machinery and equipments applications to recreation product and consumer.

Alloys 6061 is easily welded and jointed by commercial methods. The positron is one of leptons, a group of elementary particles which also included electrons, but positron is an anti - particle of electron, its mass equal $511 \mathrm{Kev} / C^{2}=$ $9.11 \times 10^{-31} \mathrm{Kg}$ with spin $1 / 2$.

It is originates from radioactive decay of artificial radioactive nuclides, e .g, $N a^{22}$ or from pair production; a gamma quantum with an energy of more than $1.022 \mathrm{Mev}$ generates a positron and an electron when encountering an atom. The annihilation of the positron with electron could be utilized to study properties of solids[1,2,3,4]. Whenpositron is injected in condensed matter, they annihilate with electrons after they are thermallized in a few picoseconds.

The trapping of the positron in defect in solids is based on the formation of an attractive potential at open volume defect, such as vacancies, and dislocations.

Corresponding author: mahmoudph8@gmail.com

DOI: 10.21608/ajnsa.2020.20124.1304

(C) Scientific Information, Documentation and Publishing Office (SIDPO)-EAEA 
The main reason for this potential is the lack of a repulsive positively charge nucleus in such defect. The sensitivity range for vacancy detection in metals starts at about one vacancy per $10^{7}$ atoms .The shape parameter or S parameter is defined as the area of the central low - momentum part of the spectrum $A_{S}$ divided by the area below the whole curve $A_{O}$ after background subtraction.

$$
\mathrm{S}=\boldsymbol{A}_{S} / \boldsymbol{A}_{\boldsymbol{O}}
$$

We can defined $\mathrm{S}$ parameter as the of number counts in the central region of annihilation line To the central region of annihilation line to the total number of counts in the peak. While wing parameter (W parameter) is taken a high momentum region far from the center, and is defined as the sum of the of counts in the wing regions of the line to the total number of counts in the peak.

\section{Experimental procedures}

In our work positron can obtained from beta decay of radioactive isotopes $\mathrm{Na}^{22}$ as follow:

$$
\mathrm{Na}^{22} \longrightarrow \mathrm{Na}^{22}+\mathrm{\beta}^{+}+\gamma
$$

The half - life of $\mathrm{Na}^{22}$ is 2.6 years and reasonable price make this isotopes the most used source in positron research. For, the simplest way to guide the positrons into is a sandwich configuration fig.(1)

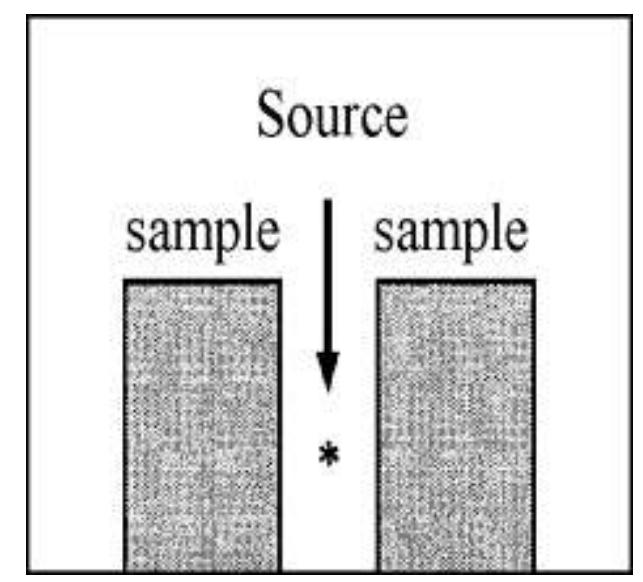

Fig. (1) Sandwich configuration of positron source

Radioactive substance was evaporated from aqueous solution of sodium chloride and deposited on thin kapton foil of $7.5 \mu \mathrm{m}$ in thickness. The energy broadening of annihilation line is measured by high resolution detectors system this system has been used in this experiment to determine the Doppler broadening parameter.

The Doppler - broadening spectrometer consists of an Ortec High Purity Germanium (HPGe) detector, with an energy resolution of $9 \times 10^{-3} \mathrm{MeV}$ for the $0.511 \mathrm{MeV}$ line of $22 \mathrm{Na}$, and an Ortec $3 \times$ $10^{-3} \mathrm{MeV}$ bias supply as shown in figure (2). The signal coming from the detector enters the input of a preamplifier and the output from the preamplifier is fed to amplifier. The output signal from the amplifier is fed to the input of an ADC to be digitized. Then the signal passes to the MCA that transfers the data to the computer system.

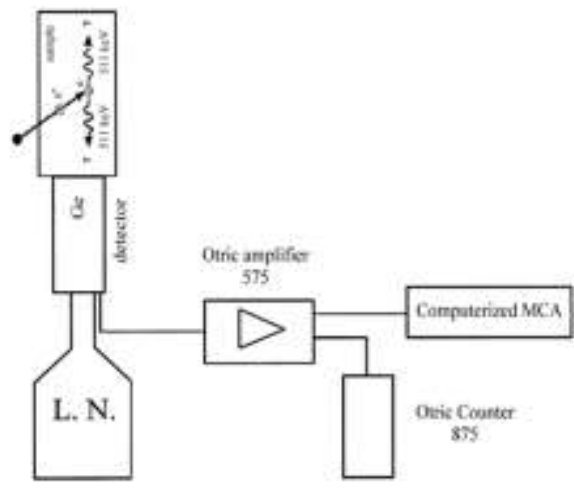

Fig. (2): Diagram to show Doppler broadening spectrometer (Ge detector)

The annihilation photons causes a charge separation that is converted by preamplifiers into

An electrical pulse, the output pulses from amplifier is fed into stabilizer to stabilize the position of the $0.511 \mathrm{Mev}$ photo peak. The output pulses from stabilizers are then fed to a multi channel analyzer.

The chemical composition of 6061 is shown in table 1 .

Table (1): shows chemical composition of $6061 \mathrm{Al}$ - alloy

\begin{tabular}{cc}
\hline Element & \%Present \\
\hline $\mathrm{Mg}$ & $0.80-1.20$ \\
$\mathrm{Si}$ & $0.40-0.80$ \\
$\mathrm{Cu}$ & $0.0-0.70$ \\
$\mathrm{Cr}$ & $0.04-0.35$ \\
$\mathrm{Zn}$ & $0.0-0.25$ \\
$\mathrm{Ti}$ & $0.0-0.15$ \\
$\mathrm{Mn}$ & $0.0-0.15$ \\
Others ( Total ) & $0.0-0.15$ \\
Others ( Each ) & $0.0-0.05$ \\
$\mathrm{Al}$ & Balance \\
\hline
\end{tabular}


$\mathrm{Al}$ - alloy 6061 were prepared in the dimensions 1.4 x 1.4 x $0.4 \mathrm{Cm}$ by using cut off machine.

The samples were polished by silicon carbide grinding papers of different sizes then etching,all samples after it rinsed in pure acetone ( $99 \%$ ), also the samples must be cleaned with water and dried perfectly. Finally all samples annealed at $420{ }^{\circ} \mathrm{C}$ for $2.30 \mathrm{~h}$.

These prepared samples were deformed at room temperature up to $22 \%$ of their original thickness using a hydraulic pressure.

\section{Results and Discussion}

Calculation the energy resolution of the system.

The energy resolution can be defined as the detector's ability to resolve the small differences in the energy of incident photons. The smaller the energy resolution the better a detector is able to distinguish between two photons of similar energy. The energy per channel (slope of linearity) was estimated to be $3.3 \times 10^{-4} \mathrm{MeV}$ per channel which multiplied by the average value of Full Width at Half Maximum of the given sources $(\mathrm{FWHM}=27.5$ channels $)$ to give the energy resolution of the system which is equal to $9 \times$ $10^{-3} \mathrm{MeV}$.

The relation between the peak position and the energy of the given sources was illustrated in figure(3).

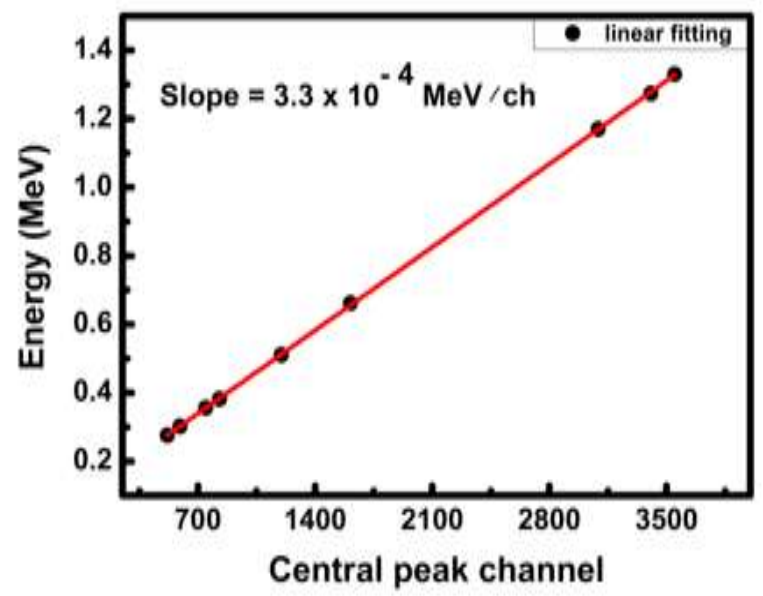

Fig. (3): The linear fit for the energy and the peak position to estimate the energy scale of the system

The energy resolution of the system decreases exponentially as a function of the energy of the given sources as shown in figure (4).

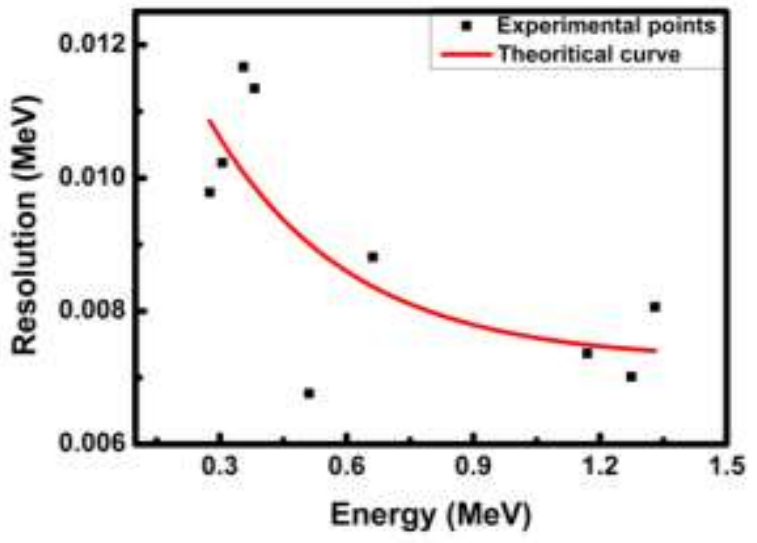

Fig. (4): the relation between resolution and the energy of the system

Calculation of positron parameters by Doppler Broadening Technique

By using the Doppler broadening annihilation radiation spectrometer we obtained the spectrum of the output pulse which represents the shift of energy from the $0.511 \mathrm{Mev}$ annihilation line.

From the spectrum, we determine the central parameter $\mathrm{S}$ which defined as the ratio of the counts in the center to the total number of counts in the peak.

The SP $16 \mathrm{k}$ software program [12] is used in determines $\mathrm{S}$ and $\mathrm{W}$ parameter automatically by canceled the background from calculation.

In Fig ( 5a) S - Parameter with thickness reduction which calculated from SP program also W Parameter in Fig (5b).

For each used sample the value of $\mathrm{S}$ parameter for the deformed sample is greater than the $S$ parameter for the annealed one, and this is because of the existence of defect due to the

deformed process and that can be clearly from Fig ( $5 \mathrm{a}$ ) as deformation increases the value of $\mathrm{S}$ parameter increases until $6 \%$ deformation then be constant which means saturation of positron trapping in opposition to the last behavior $\mathrm{W}$ parameter .

All results of positron annihilation calculated according two states trapping model[5,6]from this model $\mathrm{S}$ - parameter is defined as :

$\mathrm{S}=S_{f}\left[\frac{1+K_{t} S_{t}}{1+K_{t} S_{f}}\right]$ 
Where $S_{f}$ is $\mathrm{S}$ - parameter of annealed sample and $S_{t}$ is $\mathrm{S}$ - parameter at saturation due to trapping of positron and $K_{t}$ is a trapping rate.

We can calculate the value of $S_{t}$ From Fig ( $5 \mathrm{a}$ ) which equal ( $0.753 \pm 0.007)$ also $S_{f}(0.444 \pm$ $0.004)$.

In our work crystallite size[3] was calculated from equation (2) as follow:

$$
\mathbf{L}=\frac{\left(s_{t}-s_{f}\right)}{\left(s-s_{f}\right)} l_{d}
$$

Where $l_{d}$ is diffusion length for position which constant for aluminum $(=0.15 \mu \mathrm{m})$. The crystallite size of $6061 \mathrm{Al}$ - alloy shown in Fig.(6).

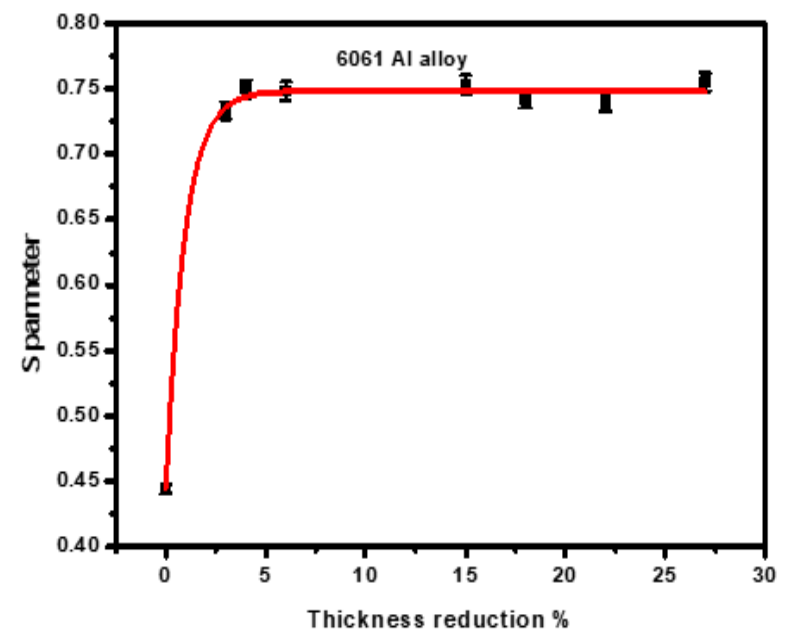

Fig. (5a): S - parameter of $6061 \mathrm{Al}$ - alloy

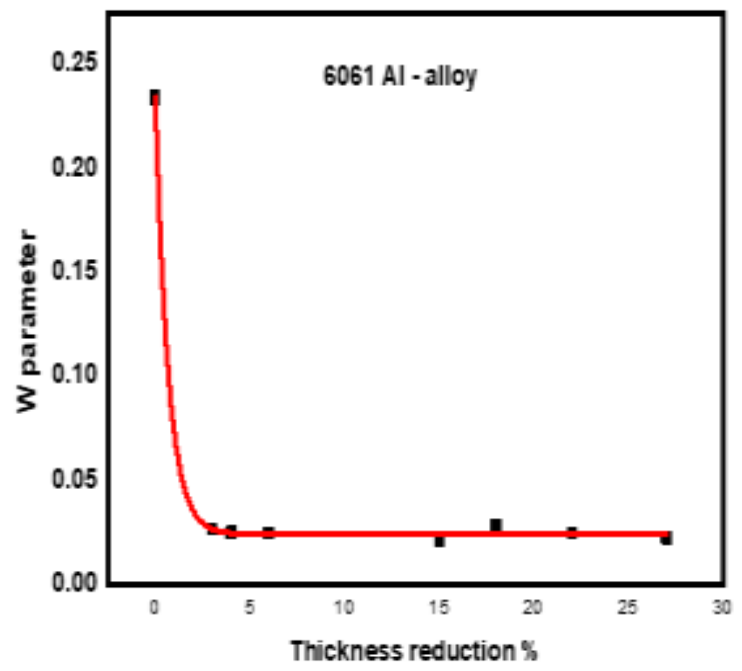

Fig. (6): Shows crystallite size of $6061 \mathrm{Al}-$ alloy
Also other parameter estimated such defect density $\left(\rho^{\prime}\right)\left(\mathrm{Cm}^{-3}\right)[8]$ which defined as number of trapping sites per unit volume by using values of trapping rate which explained in equation (4) .

$$
\rho^{\prime}=\frac{\mathrm{K}_{\mathrm{t}}}{v}
$$

Where $\mathrm{v}$ is the trapping efficiency which calculated by using trapping rate from the relation in equation ( 5 ) as follow :

$K_{t}=(1.248) \times 10^{-3}[\log (1-R)]^{2} \frac{v}{b^{3}}$

Where $\mathrm{R}$ is fractional thickness reduction. By using equation (5) at every deformation and then calculate the mean value for all we get the trapping efficiency of $6061 \mathrm{Al}-$ alloy which equal ( $1.75 \mathrm{x}$ $10^{-6} \mathrm{Cm}^{3} \mathrm{Sec}^{-1}$ ). The defect density $\rho^{\prime}$ of $6061 \mathrm{Al}$ - alloy with thickness reduction shown in Fig.(8).In this figure the defect density of $6061 \mathrm{Al}$ - alloy increases gradually till $22 \%$ then increase sharply. There a relation between defect density and dislocation density $(\rho)[8,9]$ which defined as the length defect per unit volume in equation (6) as follow:

$$
\rho^{\prime}\left(\mathrm{Cm}^{-3}\right)=\frac{\rho\left(\mathrm{Cm}^{-2}\right)}{\mathrm{b}}
$$

The dislocation density of $6061 \mathrm{Al}$ - alloy with thickness reduction was plotted in fig(9)which show that there is a exponential relation between them.

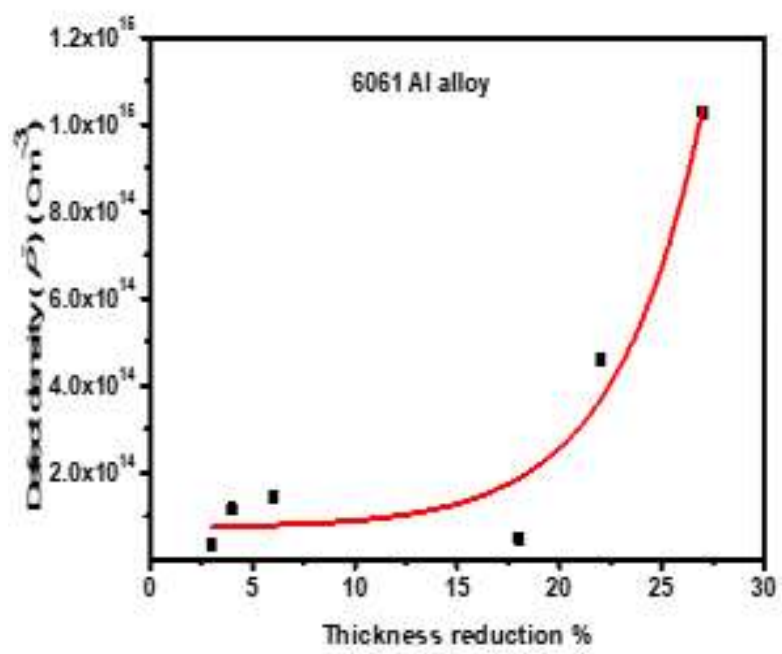

Fig. (8): defect density of $6061 \mathrm{Al}$ - alloy 


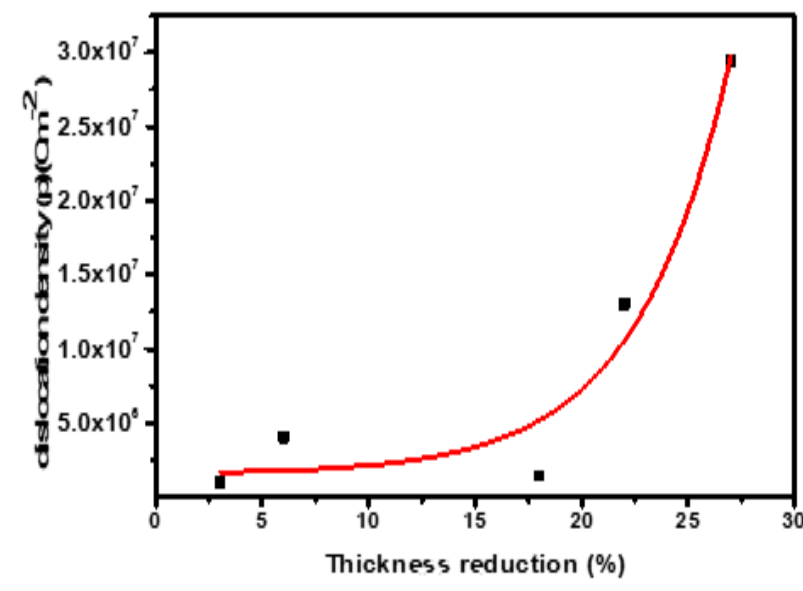

Fig. (9): dislocation density of $6061 \mathrm{Al}$ - alloy

The dislocation stored energy its amount of energy required by matter that contains defect to recovery its original states [7] . It calculated from the equation (7):

$$
\mathrm{E}\left(\mathrm{K} \mathrm{J} / m^{3}\right)=\alpha \rho \mathbf{G} \mathrm{b}^{2}
$$

Where $E$ is dislocation stored energy, $\alpha$ is dislocation interaction parameter and equal 0.5

$\rho$ is the dislocation density, $\boldsymbol{G}$ is matrix shear modulus , $b$ is the burger vector and it is equal

(2. $\left.86 \times 10^{-10} \mathrm{~m}\right) . \mathrm{G}=26 \mathrm{GPa}$. The dislocation stored energy of $6061 \mathrm{Al}$ - alloy illustrated with thickness reduction in

Fig (10).

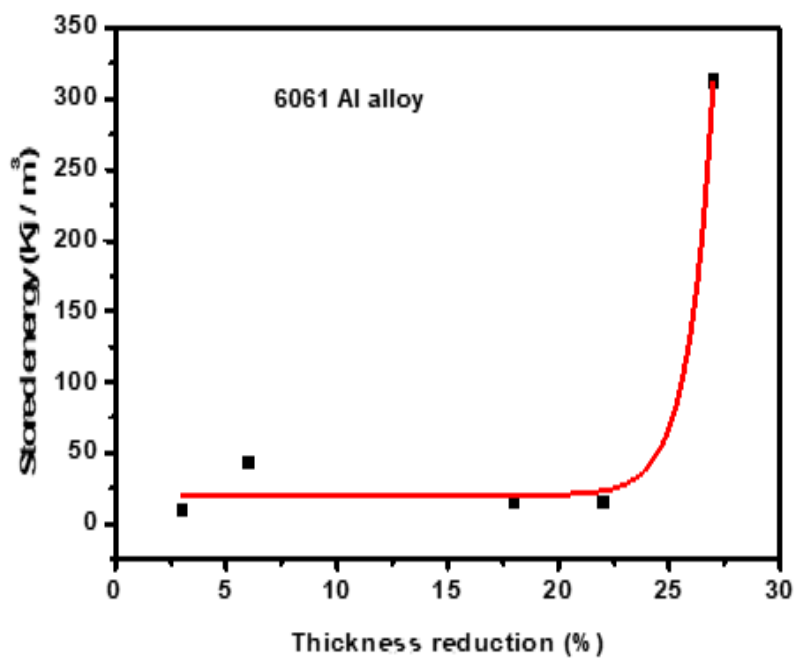

Fig. (10): Stored energy of $6061 \mathrm{Al}$ - alloy

\section{Conclusions}

According to the last measurements on the $6061 \mathrm{Al}$ - alloy by using Doppler - broadening spectroscopy the following points are conclude:

1) The $S$ - parameter is change from 0.444 at non -deformed sample to 0.149 at $27 \%$ Deformation.

2) Opposite to $S$ - Parameter behavior the $w$ decreases exponential with thickness reduction.

3) The crystallite size varies from $0.160 \mu \mathrm{m}$ at $3 \%$ deformation to $0.149 \mu \mathrm{m}$ at $27 \%$ Deformation.

4) Trapping rate of $6061 \mathrm{Al}$ - alloy increases exponentially from $6 \times 10^{7} \mathrm{Sec}^{-1}$ at $3 \%$ Deformation to18 $\times 10^{8} \mathrm{Sec}^{-1}$ at $27 \%$ deformation.

5) Defect density of our sample changes from 3.42 $\mathrm{x} 10^{13} \mathrm{Cm}^{-3}$ to $10.28 \times 10^{14} \mathrm{Cm}^{-3}$ at $3 \%$ deformation and $27 \%$ respectively.

6) Stored energy of $6061 \mathrm{Al}$ - alloy increases from 10. $39\left(\mathrm{~K} \mathrm{~J} / \mathrm{m}^{3}\right)$ of $3 \%$ deformation to 312.62 $\left(\mathrm{K} \mathrm{J} / \mathrm{m}^{3}\right)$ at $27 \%$ deformation . 


\section{References}

1. J. Baram and M.Rosen, Phys. Stat. Solidi., vol. A 16, 1973, P, 263.

2. G. Dlubek, H. Krause, R. Unger and W. Heyroth, Phys. Stat. Sol. (a) 169, R11 (1998).

3. E. A. Badawi, Surface Review and Letters, vol. 11 No. 2, 2004, P. 167.

4. R. Ferragut and A. Somoza, Phys. Stat. Sol. (a) R1 (1999).

5. B. Bergersen and M.J Stott, Solid State Commun. vol. 7, 1969, p. 1203.

6. D.C. Connors and R.N.West, Phys. Letters, vol. A 30, 1969, p. 24.

7. Abdel-Rahman, M. (2009). Detecting thermal defects in age hardening Al-6063 alloys by Positron annihilation Doppler broadening technique. Physica status solidi c, 6(11), 23592363.

8. Advanced Engineering Materials 12 (7), 559571 (2010).

9. Abdel-Rahman, M. A., Kamel, N. A., AbdelRahman, M., Abo-Elsoud, M., Lotfy, Y. A., \&Badawi, E. A. (2006). Defect Densities Using the Positron Annihilation Doppler Broadening Technique in Wrought Alloys 3003 and 3005. In Defect and Diffusion Forum (Vol. 251, pp. 79-88). Trans Tech Publications.

10. C. Dauwe, M. Dorikens, L. Dorikens and D. Segers, Appl, Phys., vol. 5, 1974, p. 117.

11. Abdel-Rahman, M. (2017). Determination of the Positron Parameters and the Stored Dislocation Energy of Plastically Deformed Wrought 3004 Al-Alloy. Int. J. New. Hor.Phys, 4(2), 43-47.

12. http://www.ifj.edu.pl/ mdryzek 